\title{
Inhalt, Vol. 7, No. 6, 1984
}

Impressum 324

Kleeberg, U. R., Hamburg-Altona

Editorial 326

Treidler, J.; Pompecki, R.; Müllerleile, U.; Garbrecht, M. und Kleeberg, U. R., Hamburg

Prognostische Aussage des Serum-CEA und therapie-

bedingte unspezifische CEA-Verläufe beim metastasie-

renden Mammakarzinom 328

Buchbesprechungen 334

Sonderbände 336

Friedmann, W.; Steffens, J. und Lobeck, H., Berlin

Immunhistochemische Diagnose des metastasierenden

Prostatakarzinoms 337

Hinweise für Autoren 341

Jehn, U. und Mezger, J. , München

Phase-II-Studie zur Behandlung der CML-Blastenkrise

mit Vindesin und Prednison 342

Buchbesprechungen 346

Aigner, K. R.; Jungbluth, A.; Link, K. H.; Walther, H.;

Midler, H.i Schwemmle, K.; Ringenberg, Th.;

Börger, G.; Ruppel, R. und Illig, L., Gießen; Voigt, H.,

Hamburg-Altona

Die isolierte hypertherme Extremitätenperfusion mit

Vindesin, Dacarbazin und Cis-Platin bei der Behandlung

maligner Melanome , 348

Buchbesprechungen 354

Neuigkeiten für die klinische Praxis 356

Görg, C., · Görg, K.; Gropp, C. und Havemann, K.,

Marburg

Aggressive Chemotherapie bei generalisiertem eosino-

philen Granulom im Erwachsenenalter 360

Wander, H.-E., Göttingen

Panmyelopathie unter Aminoglutethimid. - Ein Fall-

bericht 367

Fiebig, H. H.; Henß, H.; Arnold, H. und Löhr, G. W., Freiburg; Wellens, W.,

DuisburglRegensburg; Westerhausen, M., Duisburg und Peukert, M., Bielefeld Phase-II-Studie des wasserlöslichen Nitrosoharnstoffes ACNU bei fortgeschrittenen kolorektalen Karzinomen. . 370

Petersen, K. G.; Arnold, H.; Lehmann, M. undKerp, L., Freiburg 
Reversible orthostatische Hypotension unter Vincristin-

therapie $\quad 378$

Baczako, K. und Fischer, H. , Ulm

Das maligne metastasierende Cystosarkoma phylloides

der Mamma. - Ulmer Sammlung seltener Tumoren: VI. . 382

Onkologie-Mitteilungen 385

Jahres-Inhalt 1984389

Autorenverzeichnis $1984 \quad 392$

Contents

Imprint324

Kleeberg, U. R., Hamburg-Altona

Editorial $\quad 326$

Treidler, J.; Pompecki, R.; Müllerleile, U,; Garbrecht, M. and Kleeberg, U. R., Hamburg

Prognostic Value of Serum CEA and Therapy-Induced

Insignificant CEA Courses in Metastasized Breast Carci

noma 328

Book Reviews 334

Special Editions 336

Friedmann, W.; Steffens, J. and Lobeck, H., Berlin

Immunhistochemical Diagnosis of Metastatic Prostate

Carcinoma 337

Instructions for Authors 341

Jehn, U. and Mezger, J. , München

Phase-II Trial with Vindesine/Prednisone in the Treat

ment of CML-Blast Crisis 342

Book Reviews 346

Aigner, K. R.; Jungbluth, A.; Link, K. H.; Walther, H.; Müller, H.; Schwemmle, K.; Ringenberg, Th.; Börger, G.; Ruppel, R. and Illig, L., Gießen; Voigt, H., Hamburg-Altona

Isolated Hyperthermic Extremity Perfusion for Melanoma with Dacarbazin, Vindesine and Cis-

Platinum. . . 348

Book Reviews 354

News for Clinical Practice 356

Görg, C; Görg, K.; Gropp, C. and Havemann, K.,

Marburg

Aggressive Chemotherapy for Dissiminated Eosinophilic

Granuloma in Adults 360

Wander, H.-E., Göttingen

Pancytopenia-Induced by Aminoglutethimide. - A Case

Report 367

Fiebig, H. H.,Henß, H.; Arnold, H. and Löhr, G. W.,

Freiburg; Wellens, W. , DuisburglRegensburg;

Westerhausen, M., Duisburg and Peukert, M., Bielefeld

Phase-II Study of the Watersoluble Nitrosourea ACNU

in Advanced Colorectal Cancers 370

Petersen, K. G.; Arnold, H.; Lehmann, M. and Kerp, L.,

Freiburg 
Reversible Orthostatic Hypotension during Vincristine

Therapy

378

Baczako, K. and Fischer, H. , Ulm

Malignant Metastasizing Cystosarcoma Phylloides of the

Breast 382

Oncology Communications 385

Complete Contents $1984 \quad 389$

Author Index 1984392

Bibliographischer Hinweis: Inhaltsverzeichnisse dieser Zeitschrift erscheinen regelmäßig in current contents ${ }^{\circledR}$ sowie in anderen bibliographischen Diensten. 